\title{
Plasma C-MYC level manifesting as an indicator in progression of breast cancer
}

\author{
Weizhong Shi $i^{1,2,3}$ (D) Xiaofeng $X_{u^{1,2}}$, Ren Huang ${ }^{1,2}$, Qi $\mathrm{Yu}^{1,2}$, Peiru Zhang ${ }^{1,2}$, Suhong \\ $\mathrm{Xie}^{1,3}$, Hui Zheng ${ }^{1,3}$ \& Renquan Lu*,1,3 \\ ${ }^{1}$ Department of Clinical Laboratory, Fudan University Shanghai Cancer Center, Shanghai, PR China \\ ${ }^{2}$ Department of Clinical Laboratory, Shanghai Proton \& Heavy lon Center, Shanghai, PR China \\ ${ }^{3}$ Department of Oncology, Shanghai Medical College, Fudan University, Shanghai, PR China \\ *Author for correspondence: Tel.: +86 02164175590 ext. 82230; Fax: +86 02164175590 ext. 82204; lurenquan@126.com
}

\begin{abstract}
Aim: To investigate whether plasma C-MYC level could be an indicator in clinical progression of breast cancer. Materials \& methods: Plasma level of C-MYC expression was detected by quantitative real time PCR and the level of c-myc protein in breast cancer tissues was detected by immunohistochemistry. The expression level of C-MYC mRNA in supernatant of cancer cells culture was measured compared with the nonbreast cancer cells. Results: Plasma C-MYC level was significantly higher in patients with breast cancer than that in the controls, which associated with clinical stages, lymph node status, etc. Receiver operating characteristic curve analysis showed the sensitivity and specificity of plasma C-MYC level for diagnosis of breast cancer were 63.6 and $81.8 \%$, respectively. The expression of c-myc protein in breast cancer tissues was associated with plasma C-MYC level, even C-MYC level in supernatant of cancer cells was elevated. Conclusion: Plasma C-MYC level might be a potential indicator in progression of breast cancer.
\end{abstract}

First draft submitted: 18 February 2019; Accepted for publication: 14 May 2019; Published online: 30 May 2019

Keywords: breast cancer $\bullet C-M Y C \bullet$ circulating tumor DNA $\bullet$ diagnosis $\bullet$ indicator $\bullet$ prognosis $\bullet$ progression $\bullet$ q-PCR

Breast cancer is the most frequently diagnosed cancer and is also the leading cause of cancer death among women worldwide, accounting for almost $25 \%$ of all cancer cases and $15 \%$ of all cancer deaths [1]. The accurate and early noninvasive detection of breast cancer is a key to cure breast cancer and improves the chance of treatment successful at early stages [2]. However, traditional circulating biomarkers for breast cancer, such as CEA and CA15-3, lack sensitivity and specificity [3,4]. Therefore, there is an urgent need to search for novel noninvasive biomarkers which can facilitate the early diagnosis, prognosis and monitoring of disease progression of breast cancer.

Circulating DNA fragments released by cells are known as cell-free DNA (cfDNA), and cfDNA carrying tumorspecific genetic alterations is referred to as circulating tumor DNA (ctDNA) [5]. It has been reported that ctDNA plays an important role in tumorigenesis, progression, metastasis and therapeutic response of tumor [6-9]. However, the generative mechanism of ctDNA remains incompletely certain. Several studies demonstrate that ctDNA is derived from active tumor cellular secretions, such as exosomes, microparticles and shedding vesicles [10-12]. But other studies suggest an apoptotic origin of ctDNA from cancer cells in patients [13,14]. In recent years, with the advanced development of technology in 'liquid biopsy', ctDNA has become increasingly used as a clinical and noninvasive biomarker in the field of cancer [15-17].

Human $C-M Y C$ is located on chromosome 8q24.1 and consists of three exons and two introns, it is the member of $M Y C$ gene family which plays an important role in the regulation of cell proliferation through diverse biological function including cell cycle control, protein synthesis, apoptosis and cell adhesion [18]. Abnormal expression of $C-M Y C$ is associated with tumorigenesis and sustained tumor growth [19,20]. Several studies suggested that a higher expression of c-myc protein was observed in tissue of breast cancer and a higher level of $C-M Y C$ amplification was associated with a rapid disease recurrence and progression [21-23]. However, tissue biopsy is invasive and hard to follow-up. In the present study, we use the technique of 'liquid biopsy' to detect plasma $C-M Y C$ level in patients with primary breast cancer to investigate if plasma $C-M Y C$ level could be an indicator in progression of breast cancer. 


\section{Materials \& methods}

Study population

A total of 200 female patients diagnosed as having primary breast cancer by a clinic were recruited from August 2014 to April 2016 in Fudan University Shanghai Cancer Center. The diagnostic criteria were based on physical and imaging examinations, including blood tests, ultrasound, mammograph, computed tomography and MRI. A total of 135 cases of them including breast cancer patients $(n=110)$ and benign breast disease $(n=25)$ were eventually enrolled in our investigation according to our enrollment criteria. The exclusion criteria were patients with hemolysis $(n=4)$ or lipemia $(n=6)$, patients with incomplete medical history $(n=25)$ and nonfirst treated patients $(n=30)$. The benign breast disease included fibromas $(n=13)$, lipomas $(n=5)$, leiomyomas $(n=4)$ and other benign cysts $(n=3)$. In addition, 30 healthy women were also enrolled as the controls. All patients with primary breast cancer and patients with benign breast disease were confirmed by pathologist after surgical resection. All healthy women underwent physical and imaging examinations as breast cancer patients, to confirm that there were no related diseases. All follow-up patients underwent imaging examinations to determine whether there was recurrence or metastasis.

\section{Clinical characteristics of patients with breast cancer}

The clinical characteristics of primary breast cancer patients are shown in Supplementary Table 1. The mean age of the patients with primary breast cancer was 50.6 years; for the patients with benign breast disease, this value was 47.4 years; and 47.9 years for the healthy women. Tumor stages, grades, vascular invasion and lymph node metastasis status were assessed through pathological determination. Immunohistochemical (IHC) staining was performed by the Department of Pathology. The IHC markers included ER, PR, HER2, Ki67, EGFR, p53, Mammaglobin, AR, CK5/6 and GCDFP15. All the histological sections of tumor specimens were evaluated by two experienced pathologists.

\section{Peripheral blood samples collection \& cfDNA extraction}

The $2 \mathrm{ml}$ blood samples were drawn from a peripheral vein and collected into the ethylenediamine tetraacetic acid (EDTA)-containing tube. The blood samples were centrifuged at $1500 \times \mathrm{g}$ for $10 \mathrm{~min}$ at room temperature to separate the plasma within $2 \mathrm{~h}$. Then the supernatant was centrifugated at $12000 \times g$ for $10 \mathrm{~min}$ at room temperature to eliminate the cell debris. cfDNA was abstracted from $400 \mu \mathrm{l}$ of plasma using QIAamp DNA Blood Mini Kit (Qiagen, Germany) according to the manufacturer's instructions. The final $30 \mu$ l eluate was stored at $-80^{\circ} \mathrm{C}$ until use.

\section{DNA extraction from cell culture supernatant}

DNA in the supernatant was extracted from $400 \mu$ of growth medium using the QIAamp DNA Blood Mini Kit, according to the manufacturer's protocol. The final $30 \mu \mathrm{l}$ eluate was collected into a nuclease-free tube and stored at $-80^{\circ} \mathrm{C}$ until use.

\section{Quantitative real time PCR assay}

We designed the C-MYC gene sequence for forward primer: 5'-TCAAGAGGCGAACACACAAC-3'; reverse primer: 5'-TAACTACCTTGGGGGCCTTT-3'. We also designed the GAPDH gene [24,25] sequence as the housekeeping gene for forward primer: 5'-CTCTGCTCCTCCTGTTCGAC-3'; reverse primer: 5'ACGACCAAATCCGTTGACTC-3'. The primer was synthesized by Sangon Biotech, Inc. (Shanghai, China). The reaction system and reaction condition were optimized and confirmed by our established q-PCR assay. The reaction system was confirmed as follow: $10 \mu \mathrm{FQ}-\mathrm{PCR}$ premix kit, $0.4 \mu \mathrm{l}$ forward primer, $0.4 \mu \mathrm{l}$ reverse primer, $2 \mu \mathrm{l}$ DNA template and $7.2 \mu \mathrm{l}$ double-distilled water $\left(\mathrm{ddH}_{2} \mathrm{O}\right)$. The optimal reaction condition is denaturing at $95^{\circ} \mathrm{C}$ for $10 \mathrm{~min}$, followed by 40 cycles of $95^{\circ} \mathrm{C}$ for $15 \mathrm{~s}$ and $63^{\circ} \mathrm{C}$ for $60 \mathrm{~s}$. The reaction was carried out on the Eppendorf Mastercycler ep realplex real-time PCR system according to the manufacturer's instructions. The PCR products were identified by agarose gel electrophoresis.

\section{IHC with breast cancer tissues \& the controls}

The expression of c-myc protein was detected in 12 breast cancer tissues and 12 adjacent normal tissues by IHC, using rabbit monoclonal antibody against c-myc (ab32072, Abcam, Inc., MA, USA, 1:100 dilution) according to the manufacturers' instructions. All the results were analyzed by two independent pathologists. Intensity as well 
as the area stained was considered in the IHC scoring process. A four-level scoring system was used to evaluate the staining intensity as follows: '-', negative, score 0 ; '+', low expression, score 1; '++', moderate expression, score 2; and ' +++ ', high expression, score 3. The percentage of positive cells was divided into four levels and scored according, as were 1 (up to 25\%), 2 (25-50\%), 3 (50-75\%) and $4(75-100 \%)$. Final results were scored by multiplying the staining intensity by the percentage of positive cells scores. Final scores greater than two were considered as positive results, greater than four were considered as strong positive.

\section{Cell lines \& cell culture}

Human MDA-MB-231 cells and nonbreast cells were donated by Institute of Breast Cancer, Fudan University Shanghai Cancer Center. Cells were seeded in a 96-well plate, cultured in DMEM supplemented with 1\% penicillin/streptomycin and $10 \%$ heat-inactivated FBS. All cells were incubated at $37^{\circ} \mathrm{C}$ in a humidified atmosphere containing $5 \% \mathrm{CO}_{2}$. After $24 \mathrm{~h}$ of incubation, the growth medium was collected in nuclease-free tubes. A part of the tubes was centrifuged at $10,000 \times g$ for $10 \mathrm{~min}$, then the supernatant was transferred into new tubes and store at $-80^{\circ} \mathrm{C}$ until extraction; another part of the tubes was centrifuged at 3000 r.p.m. for $10 \mathrm{~min}$, and then the supernatant was used to detect c-myc protein by dot ELISA test. After the growth medium was absorbed, $30 \mu \mathrm{l}$ Chaps lysis buffer was added into the 96-wells plate to lyse cells on ice for $60 \mathrm{~min}$. Then lysate was also used to detected c-myc protein by dot ELISA test. The expression level of C-MYC mRNA in supernatant of the above breast cancer cells was also measured by q-PCR compared by the nonbreast cells.

\section{Dot ELISA test}

The dot ELISA test was performed to detect the c-myc protein in the culture supernatant and cell lysate of MDAMB-231 cell lines and nonbreast cells, using the method of Paller et al. [26] with some modifications. The $2 \mu$ l cell culture supernatant was dotted onto nitrocellulose membranes $(0.45 \mu \mathrm{m}$ pore size). After air drying for $30 \mathrm{~min}$, the membranes were incubated with the blocking solution (PBS with $1 \%$ BSA and $0.05 \%$ Tween-20) for $1 \mathrm{~h}$ at $37^{\circ} \mathrm{C}$ to block nonspecific binding sites.

The membranes were then washed with PBS with $0.05 \%$ Tween-20 (PBS-T) for 5 min twice. The $10 \mu \mathrm{l}$ anti-cmyc antibody (ab32072, Abcam, Inc., 1:200 dilution) was spotted. After incubation for $2 \mathrm{~h}$ at $37^{\circ} \mathrm{C}$, the membranes were washed again with PBS-T three-times to remove unbound primary antibodies. The $10 \mu$ of goat antisheep IgG was spotted. After incubation for $1 \mathrm{~h}$ at $37^{\circ} \mathrm{C}$, the membranes were washed again with PBS-T three-times to remove unbound secondary antibody. The membranes were then placed in a plate containing prepared substrate. After $15 \mathrm{~min}$, the reaction was stopped by washing the membranes three-times with distilled water. Appearance of color indicates a positive result, while the absence of color was considered a negative result. The depth of color reflects the concentration of c-myc protein.

\section{Statistical analysis}

Independent t-test and one-way ANOVA test was used to check whether there was statistic difference where applicable. Receiver operating characteristic (ROC) curve was used to illustrate the diagnostic performance of plasma C-MYC level. Kaplan-Meier method was used for survival analysis; groups were compared with log-rank test. Multivariate Cox proportional hazards regression model was used to discover predictors for breast cancer disease-free survival (DFS). The $\chi^{2}$ test was used to analyze the difference of c-myc protein expression between breast cancer tissues and adjacent normal tissues; Fisher exact test was used to analyze the difference of c-myc protein expression in the breast cancer tissues between patients with difference plasma $C-M Y C$ levels. All p-values were two sided, and those less than 0.05 were considered statistically significant. All statistical analysis was carried out using the IBM SPSS Statistics 21.0.

\section{Results}

\section{Establishment \& optimization of the determination assay for C-MYC expression levels}

The result of q-PCR amplification showed a good linear relationship from 100 to $0.01 \mathrm{ng} / \mu \mathrm{l}$. The melt curve showed the melt temperature of $C-M Y C$ was at around $84.5^{\circ} \mathrm{C}$. The PCR products were identified by agarose gel electrophoresis, and the results showed that the size of PCR amplicon of C-MYC gene was around $100 \mathrm{bp}$ (Supplementary Figure 1). 
(A)

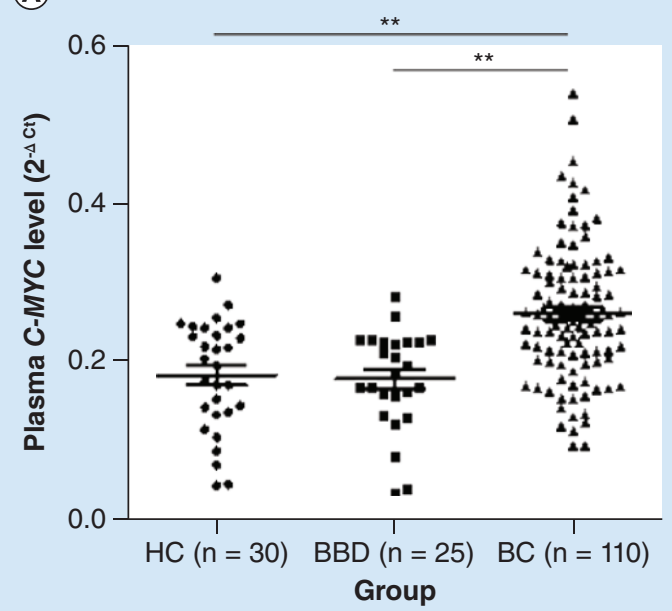

(C)

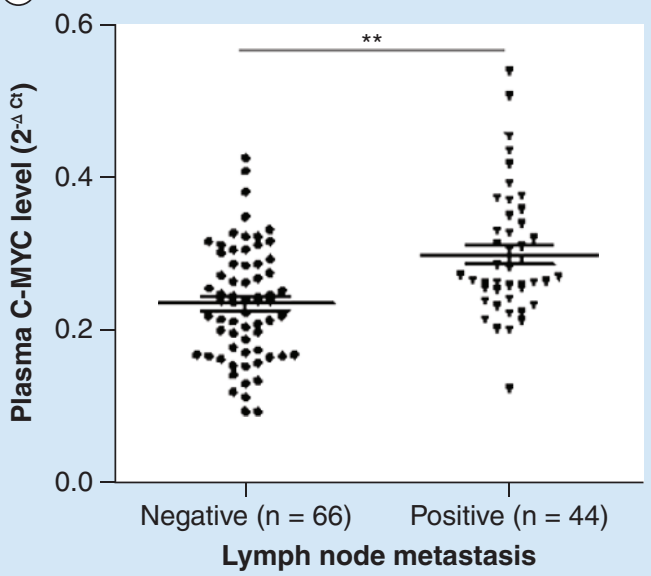

(B)

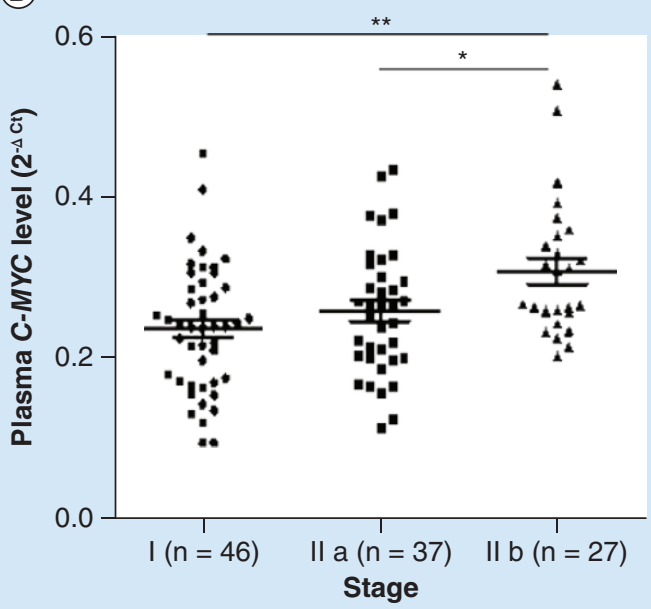

(D)

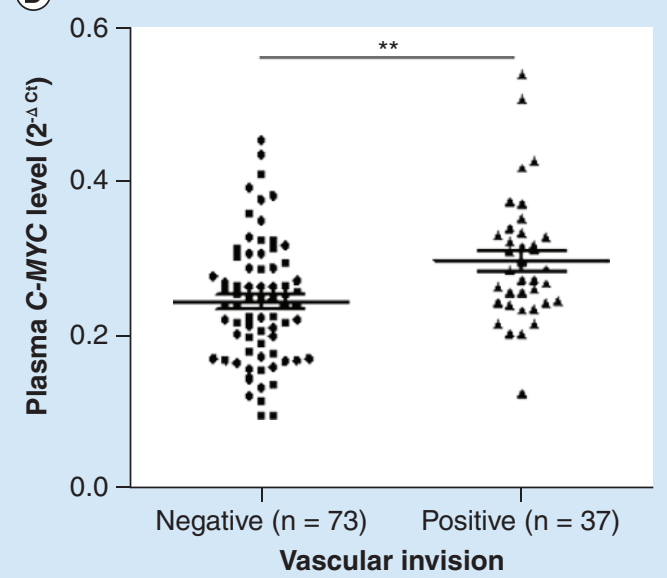

Figure 1. Association of plasma C-MYC level with clinical characteristics. (A) Plasma C-MYC level was significantly higher in BC than that in $\mathrm{HC}(\mathrm{p}<0.01)$ and BBD $(p<0.01)$; there was no significant difference of plasma C-MYC level between BBD and HC ( $p=0.78)$. (B) Plasma C-MYC levels were significantly higher in stage Ilb group than those in stage I group $(p<0.001)$ and stage Ila group $(p=0.018)$; there was no significant difference of plasma C-MYC level between stage I group and stage lla group $(p=0.209)$. (C) Plasma C-MYC level was significantly higher in lymph node positive than that in lymph node negative $(p<0.01)$. (D) Plasma C-MYC level was significantly higher in patients with positive vascular invasion than that in patients with negative vascular invasion $(p<0.01)$.

BC: Breast cancer; BBD: Benign breast disease; HC: Healthy control.

${ }^{*} \mathrm{p}<0.05 ;{ }^{* *} \mathrm{p}<0.01$.

\section{Plasma C-MYC level was elevated in the patients with breast cancer}

Plasma $C-M Y C$ level was measured in three groups. The plasma $C-M Y C$ level was calculated by a relative quantitative calculating method, referring to the method of Livak [27], the calculation formula was 'plasma $C-M Y C$ level $=2^{\wedge-c}$, $\left[C_{\mathrm{t}}=\left(C_{\mathrm{t}}\right.\right.$ value of $\left.C-M Y C\right)-\left(C_{\mathrm{t}}\right.$ value of $\left.\left.G A P D H\right)\right]$ '. We found that plasma $C-M Y C$ level in patients with primary breast cancer was significantly higher than those in patients with benign breast disease and healthy controls ( $p<0.01$, respectively); however, there was no significant difference of plasma $C-M Y C$ level between patients with benign breast disease and healthy controls $(\mathrm{p}=0.78$; Figure $1 \mathrm{~A})$.

Association of plasma C-MYC level with clinical characteristics

Plasma $C-M Y C$ level was significantly higher in patients with stage IIb group than those in stage I group $(\mathrm{p}<0.001)$ and stage IIa group $(\mathrm{p}=0.018)$. There was no significant difference of plasma $C-M Y C$ level between stage I group and stage IIa group ( $\mathrm{p}=0.209$; Figure 1B). Furthermore, plasma $C-M Y C$ level was significantly higher in patients with 
(A)

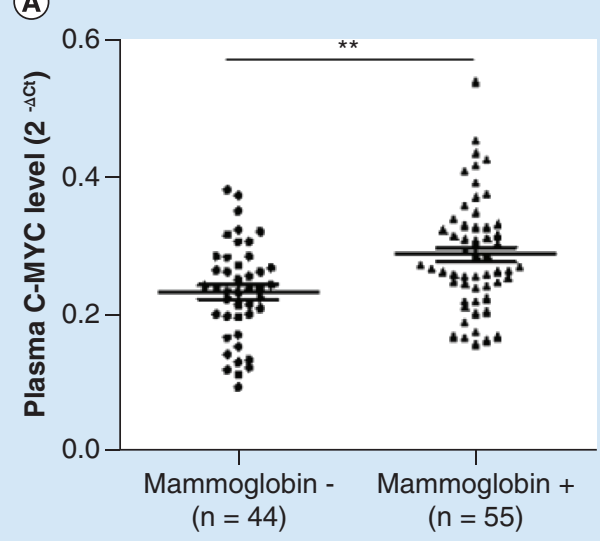

(C)

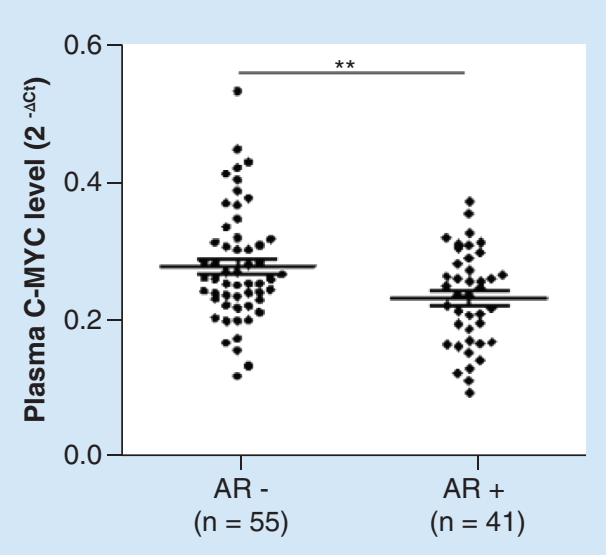

(B)

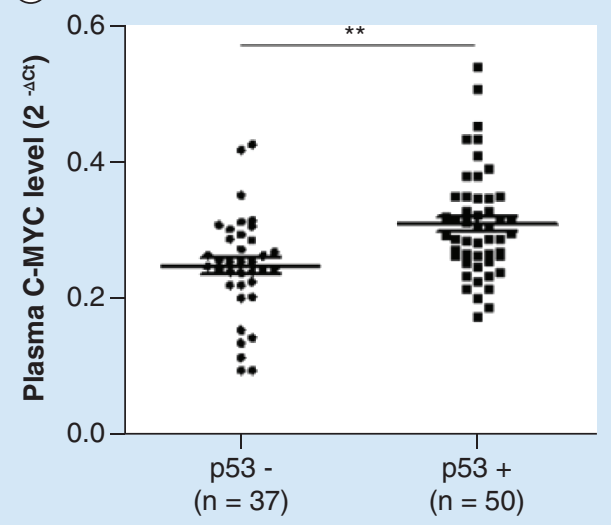

(D)

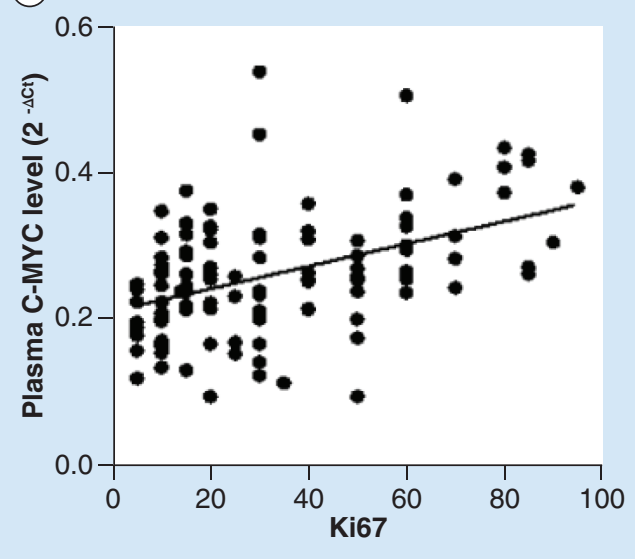

Figure 2. Correlation between plasma C-MYC level and immunohistochemical markers. (A) Correlation of plasma C-MYC level with Mammaglobin expression. Plasma C-MYC level was significantly higher in Mammaglobin ( + ) group than that in Mammaglobin (-) group ( $p=0.001)$; (B) correlation of plasma C-MYC level with p53 expression. Plasma C-MYC level was significantly higher in p53 (+) group than that in p53 (-) group (p $<0.001)$; (C) correlation of plasma C-MYC level with AR expression. Plasma C-MYC level was significantly lower in AR (+) group than that in AR (-) group $(p=0.005)$; (D) Pearson test showed a positive correlation between plasma C-MYC level and Ki67 index $(p<0.001, r=$ $0.4406)$.

$* * p<0.01$.

positive lymph node metastasis than that in patients with negative lymph node metastasis ( $\mathrm{p}<0.01$; Figure $1 \mathrm{C}$ ). Meanwhile, plasma $C-M Y C$ level was significantly higher in patients with positive vascular invasion than that in patients with negative vascular invasion $(\mathrm{p}<0.001$; Figure 1D). However, plasma $C-M Y C$ level was not correlated with age, tumor size and grade in patients with breast cancer $(\mathrm{p}=0.887,0.215$ and 0.417 , respectively).

\section{ROC curve analysis of plasma C-MYC level for the diagnosis of breast cancer}

ROC curve of plasma C-MYC level for the diagnosis of breast cancer was calculated by SPSS 21.0 software (Supplementary Figure 2). The cut-off value was determined by the best Youden index (sensitivity + specificity -1 ). When the Youden index was maximum, the cut-off value was 0.2349 , the sensitivity of the test was $63.6 \%$ (70/110), and the specificity was $81.8 \%$ (45/55). The area under the curve was 0.77 (95\% CI: $0.70-0.84)$.

\section{Correlation between plasma C-MYC level \& clinical pathological parameters}

The correlation between $C-M Y C$ level and IHC markers was analyzed by independent $\mathrm{t}$-test. We found that plasma $C-M Y C$ level was significantly higher in Mammaglobin (+) group than that in Mammaglobin (-) group ( $\mathrm{p}=0.001$; Figure 2A), a similar tendency was observed in $\mathrm{p} 53$ group $(\mathrm{p}<0.001$; Figure $2 \mathrm{~B})$. By contrary, plasma $C-M Y C$ level was significantly lower in AR ( + ) group than that in AR (-) group $(\mathrm{p}=0.005$; Figure $2 \mathrm{C})$. Moreover, Pearson 
Table 1. The 3-year disease-free survival estimated through the Kaplan-Meier methodology.

\begin{tabular}{|c|c|c|c|}
\hline \multirow[t]{2}{*}{ Factors } & \multicolumn{3}{|c|}{ DFS } \\
\hline & Mean & $95 \% \mathrm{Cl}$ & $p$-value \\
\hline Stage: & & & 0.155 \\
\hline-1 & 34.67 & $33.30-36.00$ & \\
\hline - Ila & 33.37 & $31.60-35.14$ & \\
\hline$-11 \mathrm{~b}$ & 33.91 & $32.73-35.10$ & \\
\hline Grade: & & & 0.334 \\
\hline$-I I I$ & 33.91 & $32.73-35.10$ & \\
\hline Age (years): & & & 0.198 \\
\hline$-\leq 55$ & 33.34 & $31.64-35.04$ & \\
\hline$->55$ & 34.78 & $33.07-36.49$ & \\
\hline - Negative & 34.03 & $32.49-35.58$ & \\
\hline - Positive & 33.71 & $31.88-35.58$ & \\
\hline Ki67 index: & & & $0.003^{*}$ \\
\hline$-<30 \%$ & 35.23 & $33.94-36.52$ & \\
\hline$-\geq 30 \%$ & 32.48 & $30.37-34.58$ & \\
\hline Tumor size $(\mathrm{cm})$ : & & & 0.931 \\
\hline$-\leq 2$ & 34.89 & $33.69-36.10$ & \\
\hline$->2$ & 33.17 & $31.36-34.98$ & \\
\hline Vascular invasion: & & & $<0.001^{*}$ \\
\hline - Negative & 35.49 & $34.89-36.09$ & \\
\hline
\end{tabular}

test result showed there was a correlation between plasma $C-M Y C$ level and Ki67 index $(\mathrm{p}<0.001, \mathrm{r}=0.4406$; Figure 2D). However, there was no statistical difference of plasma $C-M Y C$ levels in ER group, PR group, HER2 group, EGFR group, CK5/6 group and GCDFP15 group, respectively ( $p>0.05$, data not shown).

Role of plasma C-MYC level in prognosis of the patients with breast cancer

We divided 110 breast cancer patients from the research group into two subgroups by using the cut-off value (0.2349) calculated through ROC curve. Kaplan-Meier analysis and multivariate Cox proportional hazards regression model were used to identify important prognostic factors for DFS. Kaplan-Meier analysis revealed that plasma C-MYC level, Ki67 index and vascular invasion were significantly related to DFS ( $p=0.029,0.003$ and $<0.001$, respectively; Table 1). Patients with higher C-MYC levels had the poor outcomes by DFS analysis (Figure 3). Meanwhile, multivariate analysis revealed that vascular invasion was independent predictor for DFS ( $p=0.002$; Supplementary Table 2).

Plasma C-MYC level was positively associated with c-myc protein expression in breast cancer tissues As shown in Table 2, ten out of 12 breast cancer tissues (83.3\%) were positively stained with c-myc, and 1 out of 12 adjacent normal tissues $(8.3 \%)$ were positively stained with c-myc. The frequency of c-myc expression in breast cancer tissues was significantly higher than in adjacent normal tissues $(\mathrm{p}<0.001)$. 


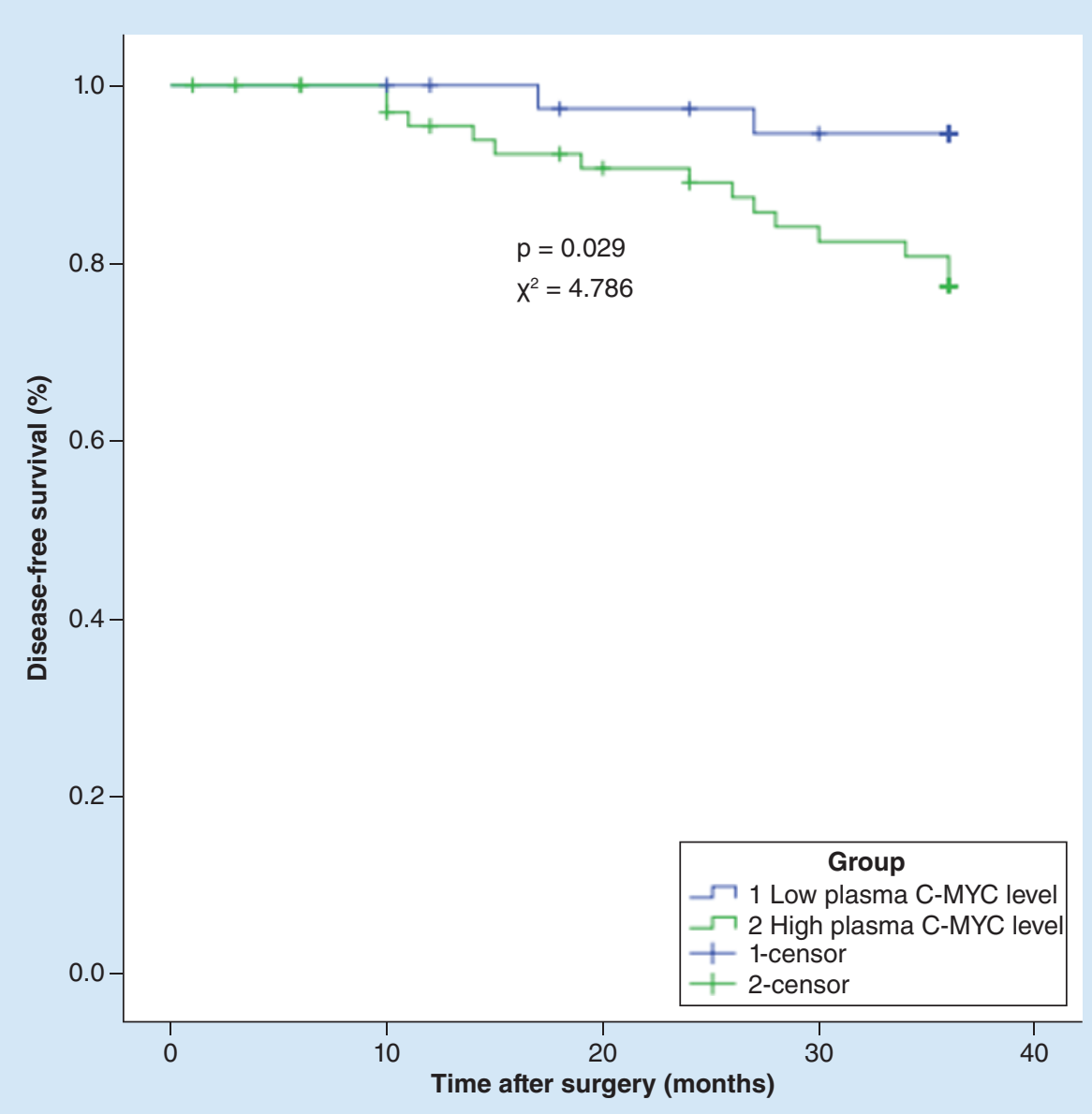

Figure 3. The 3-year disease-free survival of high and low plasma C-MYC level in breast cancer patients. Breast cancer patients with higher C-MYC levels had worse outcomes by disease-free survival analysis $(p=0.029)$.

\section{Table 2. Expression of c-myc protein in different groups by immunohistochemical staining.}

\begin{tabular}{|llll|}
\hline & & Expression of c-myc & p-value \\
\cline { 2 - 3 } & Negative $($ IHC score $<2)$ & Positive $($ IHC score $\geq 2)$ & $<0.001^{\dagger}$ \\
\hline Control & 2 & 10 & \\
\hline${ }^{*} p<0.01$. & 11 & 1 & \\
$B C:$ Breast cancer; IHC: Immunohistochemical. & & \\
\hline
\end{tabular}

\begin{tabular}{|c|c|c|c|}
\hline \multirow[t]{2}{*}{ Groups } & \multicolumn{2}{|c|}{ Expression of c-myc } & \multirow[t]{2}{*}{ p-value } \\
\hline & Weak positive/negative (IHC score $<4$ ) & Strong positive (IHC score $\geq 4)$ & \\
\hline High C-MYC level & 0 & 8 & \\
\hline
\end{tabular}

As for the relationships the protein levels of c-myc in the cancer tissues and C-MYC levels in ctDNA of the patients with breast cancer, we found that the frequency of high c-myc expression in patients with high plasma $C-M Y C$ level was significantly higher than in low plasma $C-M Y C$ level $(\mathrm{p}=0.018$; Table 3$)$. Furthermore, the expression of c-myc protein was higher in patients with higher plasma $C-M Y C$ levels (Figure 4). 

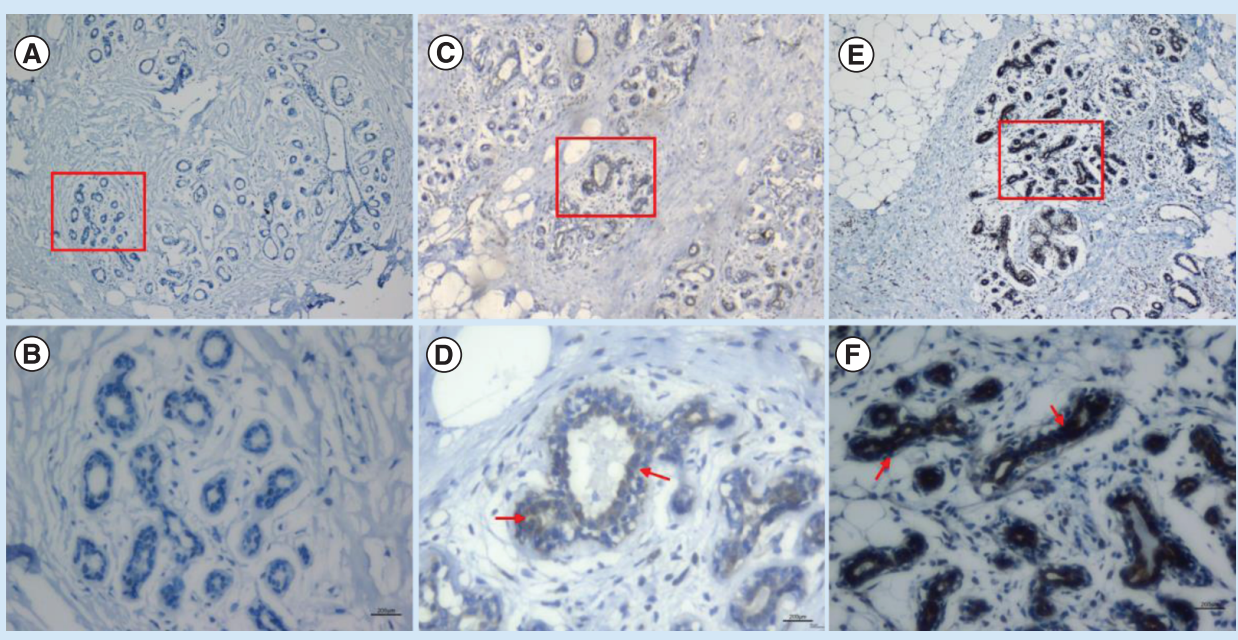

Figure 4. Immunohistochemical staining of c-myc in breast cancer tissue. (A \& B) Negative expression of c-myc in adjacent normal tissues ( $\times 100$ and $\times 400$ magnification, respectively). (C \& D) Low expression of c-myc in breast cancer tissues of patients with low plasma C-MYC level (x100 and x400 magnification, respectively). (E \& F) High expression of c-myc in breast cancer tissues of patients with high plasma C-MYC level ( $\times 100$ and $\times 400$ magnification, respectively).

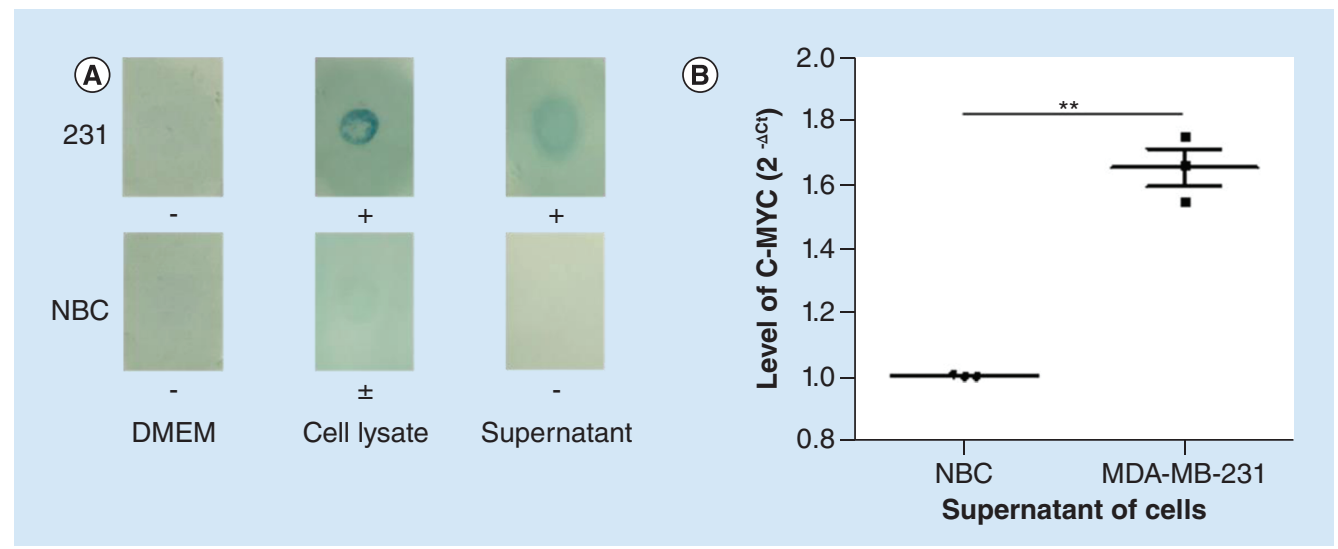

Figure 5. Comparison of c-myc protein expression and C-MYC level in MDA-MB-231 cells with nonbreast cancer cells. (A) A positive expression of c-myc protein in lysate and culture supernatant of MDA-MB-231 cells. The depth of color indicated that the concentration of c-myc protein in cell lysate was higher than that in culture supernatant. Meanwhile, the lysate of nonbreast cancer cells showed a weak positive expression of c-myc protein, and the culture supernatant showed a negative result. (B) The expression levels of C-MYC mRNA in supernatant of MDA-MB-231 cells were higher than those of the nonbreast controls $(p<0.001)$.

$* * \mathrm{p}<0.01$.

Positive expression of c-myc protein by dot ELISA test

The dot ELISA test results showed a positive expression of c-myc protein in lysate and culture supernatant of MDA-MB-231 cells. The depth of color indicated that the concentration of c-myc protein in cell lysate was higher than that in culture supernatant. Meanwhile, the lysate of nonbreast cancer cells showed a weak positive expression of c-myc protein, and the culture supernatant showed a negative result (Figure 5A).

\section{Comparing C-MYC level in supernatant of breast cancer cells with nonbreast cancer cells}

As well, the expression levels of $C-M Y C$ mRNA in supernatant of MDA-MB-231 cells were higher than those of the nonbreast controls $(\mathrm{p}<0.001)$, as were shown in Figure 5B. 


\section{Discussion}

Breast cancer is one of the most common cancer among women worldwide, both in term of incidence and mortality [28]. An accurate and early noninvasive biomarker is critical for the treatment and prognosis of breast cancer. Recently, research on the role of ctDNA involved tumorigenesis and cancer progression has grown exponentially [9,29]. Several studies have suggested that ctDNA is a potential biomarker of various types of cancers [30-32].

In this study, we performed the determination of $C-M Y C$ level in plasma. Our results showed that plasma $C-M Y C$ level in the patients with primary breast cancer was significantly higher than in the patients with benign breast disease and healthy controls. The $C-M Y C$ proto-oncogene belongs to the $M Y C$ family and is commonly activated in tumorigenesis. It is associated with cell proliferation and tends to be highly expressed in most rapidly proliferating cells [33,34]. Our study showed a consistent result with Escot [21] who first reported C-MYC gene abnormalities in primary breast cancer. However, Ravaioli et al. [35] reported that there was no statistical difference of cfDNA quantity and integrity of $M Y C$ between breast cancer patients and healthy controls in their research. We speculate that the differences may be due to the diversity in population or race.

Furthermore, we divided the patients with primary breast cancer into different subgroups according to their clinical characteristics, including age, tumor size, stages, grades, lymph node metastasis status and vascular invasion status. We found that patients with higher stage had higher plasma $C-M Y C$ level than those with lower stage. It indicated that plasma $C-M Y C$ level was related to the progression of breast cancer. However, since no more higher stage breast cancer patients was enrolled in this study, we were unable to compared plasma $C-M Y C$ level in patients with more advanced breast cancer. Furthermore, we also found that plasma $C-M Y C$ level in patients with vascular invasion positive was significantly higher than that in patients with vascular invasion negative; the same tendency was shown in the patients with positive lymph node metastasis. Vascular invasion and lymph node metastasis status have been considered as indicators of cancer progression and prognostic factors for breast cancer [36,37]. It has been reported that patients with lymph node metastasis were faced with a poor prognosis and a higher risk of relapse [38]. Thus, our results also indicated that the increased plasma $C-M Y C$ level maybe also be associated with a poor prognosis of breast cancer.

For a further investigation, the correlation between plasma $C-M Y C$ level and IHC markers was analyzed. The IHC markers include ER, PR, HER2, Ki67, Mammaglobin, p53, EGFR, AR, CK5/6 and GCDFP15. The results showed that plasma $C-M Y C$ level in Mammaglobin (+) group was significantly higher than that in Mammaglobin (-) group, a similar tendency was shown in p53 group, whereas the plasma $C-M Y C$ level was significantly lower in $\mathrm{AR}(+)$ group than in $\mathrm{AR}(-)$ group. It has been reported that positive expressions of p53 [39,40], EGFR [41-43], Mammaglobin [2829] and CK5/6 [23,30] are related to a poor prognosis of breast cancer, whereas positive expressions of AR [39,44] and GCDFP15 [45,46] are related to a favorable prognosis. Although there was no statistical difference in CK5/6, EGFR and GCDFP15 group, the results showed a higher level in CK5/6 (+) group than CK5/6 (-) group, as well as EGFR group, and a lower $C-M Y C$ level in GCDFP15 (+) group than that in GCDFP15 (-) group (data not shown). Moreover, our results also showed a positive correlation between plasma $C-M Y C$ level and Ki67 index. It has been reported that the protein Ki67 is expressed in the nucleus of cycling cells and used to measure the activity of cell proliferation [47-49]. Ki67 index is recognized as an independent prognostic factor in breast cancer [50-54], high Ki67 index is associated with a poor prognosis in breast cancer. These evidences further indicated that higher plasma $C-M Y C$ level might be associated with a poor prognosis of breast cancer.

To further confirm that plasma $C-M Y C$ level could be a prognostic factor for breast cancer, we followed-up 110 breast cancer patients for 3 years. Up to the end of follow-up, 16 patients had recurrence or metastasis, and 14 patients were lost to follow-up. Kaplan-Meier survival analysis showed that higher plasma $C-M Y C$ level was related with shortened 3-year DFS. This is partially consistent with Wang's finding that patients with positive expression of c-myc protein exhibited a shortened DFS without tumor with an increasing recurrence rate and lower survival rate [55]. Therefore, we speculated that there was a correlation between plasma $C-M Y C$ level and c-myc protein expression in breast cancer tissues.

As a validation, we detected the c-myc protein expression both in breast cancer tissues and adjacent normal tissues by IHC. Interestingly, we found that the positive rate of c-myc protein expression in breast cancer tissues was significantly higher than that in adjacent normal tissues $(\mathrm{p}<0.05)$. Furthermore, we also found that plasma $C-M Y C$ levels in breast cancer patients were positively associated with the expression of c-myc protein in breast cancer tissues. 
In addition, we detected c-myc protein in the culture supernatant and lysate of MDA-MB-231 breast cancer cells by dot ELISA test. The results showed that a positive expression of c-myc protein was both in culture supernatant and lysate of cells. Meanwhile, we abstracted cfDNA from the culture supernatant of MDA-MB-231 breast cancer cells and then detected $C-M Y C$ level by q-PCR assay. We found that $C-M Y C$ level in supernatant of breast cancer cells was significantly higher than in nonbreast cancer. Bronkhorst $e$ t al. [10] have reported that the occurrence of cfDNA was primarily a result of actively secreted DNA, perhaps in association with a protein complex. Wang et al. [12] also found that cells could release cfDNA actively via exosomes. Therefore, our results also suggest that plasma $C-M Y C$ may be secreted into peripheral blood by breast cancer cells.

However, our study still has some limitations. First, we used a small sample size in this study; therefore, a study with a larger sample size is required to verify the results. Second, the number of patients with recurrence or metastasis was too small, which may lead to statistical deviation. Thus, a longer follow-up is also needed to evaluate the relationship between plasma $C-M Y C$ level and DFS or overall survival. Finally, we only analyzed the $C-M Y C$ gene in the present study. Therefore, to strengthen the study, we will analyze other cancer-irrelevant genes to demonstrate the specificity of increased plasma $C-M Y C$ level in breast cancer patients in our future research.

\section{Conclusion}

In conclusion, our study suggests that plasma $C-M Y C$ level might be a potential indicator in progression of breast cancer, which as a part of liquid biopsy will be very promising in clinical application.

\section{Summary points}

- Plasma C-MYC level was significantly higher in patients with primary breast cancer than that in patients with benign breast disease and healthy controls.

- Plasma C-MYC level was associated with tumor stages, Ki67 index, lymph node metastasis status, vascular invasion status and expression of Mammaglobin, p53 and AR.

- Kaplan-Meier analysis revealed that plasma C-MYC level, Ki67 index and vascular invasion were significantly related to disease-free survival.

- The frequency of c-myc expression in breast cancer tissues was significantly higher than in adjacent normal tissues. And the expression of c-myc protein was higher in patients with higher plasma C-MYC levels.

- A positive expression of c-myc protein in lysate and culture supernatant of breast cancer cells, and concentration of c-myc protein in cell lysate was higher than that in culture supernatant.

- The expression levels of C-MYC mRNA in supernatant of breast cancer cells were higher than those of the nonbreast controls.

- Plasma C-MYC level might be a potential indicator in progression of breast cancer, which as a part of liquid biopsy will be very promising in clinical application.

Supplementary data

To view the supplementary data that accompany this paper please visit the journal website at: www.futuremedicine.com/doi/full/ 10.2217/bmm-2019-0073

Author contributions

WZ Shi and RQ Lu conceived experiments and wrote the manuscript. WZ Shi, R Huang, Q Yu and PR Zhang performed experiments. $\mathrm{XF} \mathrm{Xu}, \mathrm{H}$ Zheng and $\mathrm{SH}$ Xie prepared tables and figures. All authors discussed the results and commented on the manuscript.

Financial \& competing interests disclosure

This work is partially supported by the National Natural Science Foundation of China (grant nos. NSF-81572552, NSF-81402352, NSF-81772774 and NSF-81772808), Shanghai Science and Technology Committee Sponsorship (grant no. KW17411963500). The authors have no other relevant affiliations or financial involvement with any organization or entity with a financial interest in or financial conflict with the subject matter or materials discussed in the manuscript apart from those disclosed.

No writing assistance was utilized in the production of this manuscript. 


\section{Ethical conduct of research}

The authors state that the study has been approved by Ethics Committee of Fudan University Shanghai Cancer Center. Also, they have followed the principles in the Declaration of Helsinki. In addition, all participants enrolled in this study have been given written informed consent.

\section{Open access}

This work is licensed under the Attribution-NonCommercial-NoDerivatives 4.0 Unported License. To view a copy of this license, visit http://creativecommons.org/licenses/by-nc-nd/4.0/

\section{Data sharing statement}

All of the individual participant data collected during the trial will be available.

\section{References}

Papers of special note have been highlighted as: $\bullet$ of interest; $\bullet \bullet$ of considerable interest

1. Bray F, Ferlay J, Soerjomataram I, Siegel RL, Torre LA, Jemal A. Global cancer statistics 2018: GLOBOCAN estimates of incidence and mortality worldwide for 36 cancers in 185 countries. CA Cancer J. Clin. 68(6), 394-424 (2018).

2. Shamsi M, Pirayesh IJ. Breast cancer: early diagnosis and effective treatment by drug delivery tracing. Nucl. Med. Rev. Cent. East Eur. 20(1), 45-48 (2017).

3. Hanash SM, Baik CS, Kallioniemi O. Emerging molecular biomarkers - blood-based strategies to detect and monitor cancer. Nat. Rev. Clin. Oncol. 8(3), 142-150 (2011).

4. Duffy MJ, Evoy D, McDermott EW. CA 15-3: uses and limitation as a biomarker for breast cancer. Clin. Chim. Acta 411(23-24), 1869-1874 (2010).

5. Schwarzenbach H, Hoon DS, Pantel K. Cell-free nucleic acids as biomarkers in cancer patients. Nat. Rev. Cancer 11(6), 426-437 (2011).

6. Fribbens C, Garcia MI, Beaney M et al. Tracking evolution of aromatase inhibitor resistance with circulating tumour DNA analysis in metastatic breast cancer. Ann. Oncol. 29(1), 145-153 (2018).

7. Ma F, Zhu W, Guan Y et al. ctDNA dynamics: a novel indicator to track resistance in metastatic breast cancer treated with anti-HER2 therapy. Oncotarget 7(40), 66020-66031 (2016).

8. Kato K, Uchida J, Kukita Y et al. Numerical indices based on circulating tumor DNA for the evaluation of therapeutic response and disease progression in lung cancer patients. Sci. Rep. 6, 29093 (2016).

9. Cheng F, Su L, Qian C. Circulating tumor DNA: a promising biomarker in the liquid biopsy of cancer. Oncotarget 7(30), 48832-48841 (2016).

10. Bronkhorst AJ, Wentzel JF, Aucamp J, van Dyk E, du Plessis L, Pretorius PJ. Characterization of the cell-free DNA released by cultured cancer cells. Biochim. Biophys. Acta 1863(1), 157-165 (2016).

11. Burnham P, Kim MS, Agbor-Enoh S et al. Single-stranded DNA library preparation uncovers the origin and diversity of ultrashort cell-free DNA in plasma. Sci. Rep. 6, 27859 (2016).

12. Wang W, Kong P, Ma G et al. Characterization of the release and biological significance of cell-free DNA from breast cancer cell lines. Oncotarget 8(26), 43180-43191 (2017).

-• Demonstrates that cell-free DNA (cfDNA) is released from breast cancer mainly by active secretion.

13. Ershova ES, Jestkova EM, Chestkov IV et al. Quantification of cell-free DNA in blood plasma and DNA damage degree in lymphocytes to evaluate dysregulation of apoptosis in schizophrenia patients. J. Psychiatr. Res. 87, 15-22 (2017).

14. Tost J. Follow the trace of death: methylation analysis of cell-free DNA for clinical applications in non-cancerous diseases. Epigenomics 8(9), 1169-1172 (2016).

15. Reinert T, Scholer LV, Thomsen R et al. Analysis of circulating tumour DNA to monitor disease burden following colorectal cancer surgery. Gut 65(4), 625-634 (2016).

16. Szpechcinski A, Chorostowska-Wynimko J, Struniawski R et al. Cell-free DNA levels in plasma of patients with non-small-cell lung cancer and inflammatory lung disease. Br. J. Cancer 113(3), 476-483 (2015).

17. Dawson SJ, Tsui DW, Murtaza M et al. Analysis of circulating tumor DNA to monitor metastatic breast cancer. N. Engl. J. Med. 368(13), 1199-1209 (2013).

-. Demonstrates that circulating tumor DNA is an informative, inherently specific and highly sensitive biomarker of metastatic breast cancer.

18. Dang CV. c-Myc target genes involved in cell growth, apoptosis, and metabolism. Mol. Cell Biol. 19(1), 1-11 (1999).

19. Pelengaris S, Khan M, Evan G. c-MYC: more than just a matter of life and death. Nat. Rev. Cancer 2(10), $764-776$ (2002).

20. Nesbit CE, Tersak JM, Prochownik EV. MYC oncogenes and human neoplastic disease. Oncogene 18(19), 3004-3016 (1999). 
21. Escot $\mathrm{C}$, Theillet $\mathrm{C}$, Lidereau $\mathrm{R}$ et al. Genetic alteration of the c-myc protooncogene $(M Y C)$ in human primary breast carcinomas. Proc. Natl Acad. Sci. USA 83(13), 4834-4838 (1986).

22. Cline MJ, Battifora H, Yokota J. Proto-oncogene abnormalities in human breast cancer: correlations with anatomic features and clinical course of disease. J. Clin. Oncol. 5(7), 999-1006 (1987).

23. Pertschuk LP, Feldman JG, Kim DS et al. Steroid hormone receptor immunohistochemistry and amplification of c-myc protooncogene. Relationship to disease-free survival in breast cancer. Cancer 71(1), 162-171 (1993).

24. Karatayli E, Altunoglu YC, Karatayli SC, Yurdaydin C, Bozdayi AM. Free circulating nucleic acids in plasma and serum as a novel approach to the use of internal controls in real time PCR based detection. J. Virol. Methods 207, 133-137 (2014).

25. Chen W, Cai F, Zhang B, Zhong XY. Strategies of reducing input sample volume for extracting circulating cell-free nuclear DNA and mitochondrial DNA in plasma. Clin. Chem. Lab. Med. 50(2), 261-265 (2011).

26. Paller V, Besana CM, Valdez I. Dot enzyme-linked immunosorbent assay (ELISA) for the detection of Toxocara infection using a rat model. J. Parasit. Dis. 41(4), 933-939 (2017).

- Reference method of dot ELISA test in our research.

27. Livak KJ, Schmittgen TD. Analysis of relative gene expression data using real-time quantitative PCR and the 2(-Delta Delta C([T]) method. Methods 25(4), 402-408 (2001).

28. DeSantis CE, Ma J, Goding SA, Newman LA, Jemal A. Breast cancer statistics, 2017, racial disparity in mortality by state. CA Cancer J. Clin. 67(6), 439-448 (2017).

29. Bronkhorst AJ, Wentzel JF, Aucamp J, van Dyk E, du Plessis L, Pretorius PJ. Characterization of the cell-free DNA released by cultured cancer cells. Biochim. Biophys. Acta 1863(1), 157-165 (2016).

- Reveals that the occurrence of cfDNA was primarily a result of actively secreted DNA, perhaps in association with a protein complex.

30. Valpione S, Gremel G, Mundra P et al. Plasma total cell-free DNA (cfDNA) is a surrogate biomarker for tumour burden and a prognostic biomarker for survival in metastatic melanoma patients. Eur. J. Cancer 88, 1-9 (2018).

31. Bhangu JS, Taghizadeh H, Braunschmid T, Bachleitner-Hofmann T, Mannhalter C. Circulating cell-free DNA in plasma of colorectal cancer patients - a potential biomarker for tumor burden. Surg. Oncol. 26(4), 395-401 (2017).

32. Ai B, Liu H, Huang Y, Peng P. Circulating cell-free DNA as a prognostic and predictive biomarker in non-small cell lung cancer. Oncotarget 7(28), 44583-44595 (2016).

33. Nisen PD, Zimmerman KA, Cotter SV, Gilbert F, Alt FW. Enhanced expression of the $N$-myc gene in Wilms' tumors. Cancer Res. 46(12 Pt 1), 6217-6222 (1986).

34. Sawczuk IS, Olsson CA, Buttyan R et al. Gene expression in renal growth and regrowth. J. Urol. 140(5 Pt 2), 1145-1148 (1988).

35. Maltoni R, Casadio V, Ravaioli S et al. Cell-free DNA detected by 'liquid biopsy' as a potential prognostic biomarker in early breast cancer. Oncotarget 8(10), 16642-16649 (2017).

-. Suggests that cfDNA detected as liquid biopsy could have great potential in clinical practice.

36. Rezaianzadeh A, Talei A, Rajaeefard A et al. Vascular invasion as an independent prognostic factor in lymph node negative invasive breast cancer. Asian Pac. J. Cancer Prev. 13(11), 5767-5772 (2012).

37. Malfettone A, Saponaro C, Paradiso A, Simone G, Mangia A. Peritumoral vascular invasion and NHERF1 expression define an immunophenotype of grade 2 invasive breast cancer associated with poor prognosis. Bmc Cancer 12, 106 (2012).

38. Roxanis I, Colling R, Kartsonaki C, Green AR, Rakha EA. The significance of tumour microarchitectural features in breast cancer prognosis: a digital image analysis. Breast Cancer Res. 20(1), 11 (2018).

39. Maeda T, Nakanishi $Y$, Hirotani $Y$ et al. Immunohistochemical co-expression status of cytokeratin $5 / 6$, androgen receptor, and $\mathrm{p} 53$ as prognostic factors of adjuvant chemotherapy for triple negative breast cancer. Med. Mol. Morphol. 49(1), 11-21 (2016).

40. Biesaga B, Niemiec J, Ziobro M. Microvessel density and status of $\mathrm{p} 53$ protein as potential prognostic factors for adjuvant anthracycline chemotherapy in retrospective analysis of early breast cancer patients group. Pathol. Oncol. Res. 18(4), 949-960 (2012).

41. Gonzalez-Conchas GA, Rodriguez-Romo L, Hernandez-Barajas D et al. Epidermal growth factor receptor overexpression and outcomes in early breast cancer: a systematic review and a meta-analysis. Cancer Treat. Rev. 62, 1-8 (2018).

42. Tsutsui S, Ohno S, Murakami S, Hachitanda Y, Oda S. Prognostic value of epidermal growth factor receptor (EGFR) and its relationship to the estrogen receptor status in 1029 patients with breast cancer. Breast Cancer Res. Treat. 71(1), 67-75 (2002).

43. Zhang $M$, Zhang X, Zhao $S$ et al. Prognostic value of survivin and EGFR protein expression in triple-negative breast cancer (TNBC) patients. Target Oncol. 9(4), 349-357 (2014).

44. Adamo B, Ricciardi G, Ieni A et al. The prognostic significance of combined androgen receptor, E-Cadherin, Ki67 and CK5/6 expression in patients with triple negative breast cancer. Oncotarget 8(44), 76974-76986 (2017).

45. Luo MH, Huang YH, Ni YB et al. Expression of mammaglobin and gross cystic disease fluid protein-15 in breast carcinomas. Hum. Pathol. 44(7), 1241-1250 (2013). 
46. Darb-Esfahani S, von Minckwitz G, Denkert C et al. Gross cystic disease fluid protein 15 (GCDFP-15) expression in breast cancer subtypes. BMC Cancer 14, 546 (2014).

47. Woldemeskel M, Hawkins I, Whittington L. Ki-67 protein expression and tumor associated inflammatory cells (macrophages and mast cells) in canine colorectal carcinoma. BMC Vet. Res. 13(1), 111 (2017).

48. Carbognin L, Sperduti I, Fabi A et al. Prognostic impact of proliferation for resected early stage 'pure' invasive lobular breast cancer: cut-off analysis of Ki67 according to histology and clinical validation. Breast 35, 21-26 (2017).

49. Pascale M, Aversa C, Barbazza R et al. The proliferation marker Ki67, but not neuroendocrine expression, is an independent factor in the prediction of prognosis of primary prostate cancer patients. Radiol. Oncol. 50(3), 313-320 (2016).

50. Dowsett M, Nielsen TO, A'Hern R et al. Assessment of Ki67 in breast cancer: recommendations from the International Ki67 in Breast Cancer working group. J. Natl Cancer Inst. 103(22), 1656-1664 (2011).

51. Tashima R, Nishimura R, Osako T et al. Evaluation of an optimal cut-off point for the Ki-67 index as a prognostic factor in primary breast cancer: a retrospective study. PLoS ONE 10(7), e119565 (2015).

52. Sahin AA, Ro J, Ro JY et al. Ki-67 immunostaining in node-negative stage I/II breast carcinoma. Significant correlation with prognosis. Cancer 68(3), 549-557 (1991).

53. Yerushalmi R, Woods R, Ravdin PM, Hayes MM, Gelmon KA. Ki67 in breast cancer: prognostic and predictive potential. Lancet Oncol. 11(2), 174-183 (2010).

54. Inwald EC, Klinkhammer-Schalke M, Hofstadter $\mathrm{F}$ et al. Ki-67 is a prognostic parameter in breast cancer patients: results of a large population-based cohort of a cancer registry. Breast Cancer Res. Treat. 139(2), 539-552 (2013).

55. Wang J, Li M, Chen D et al. Expression of C-myc and beta-catenin and their correlation in triple negative breast cancer. Minerva Med. 108(6), 513-517 (2017).

- Reveals that the positive expression of $\mathrm{C}$-myc and $\beta$-catenin in triple-negative breast cancer tissue might be closely correlated with clinical features of cancer and the survival prognosis. 
